\title{
A novel microRNA signature for pathological grading in lung adenocarcinoma based on TCGA and GEO data
}

\author{
ZHIYU YANG $^{1 *}$, HONGKUN YIN $^{2 *}$, LEI SHI $^{3}$ and XIAOHUA QIAN ${ }^{1}$ \\ ${ }^{1}$ SJTU-Yitu Joint Laboratory of Artificial Intelligence in Healthcare, School of Biomedical Engineering, \\ Shanghai Jiao Tong University, Shanghai 200030; ${ }^{2}$ Shanghai Yitu Healthcare Technology Co. Ltd., \\ Shanghai 200051; ${ }^{3}$ Hangzhou Yitu Healthcare Technology Co. Ltd., Hangzhou, Zhejiang 310012, P.R. China
}

Received September 14, 2019; Accepted February 11, 2020

DOI: $10.3892 / \mathrm{ijmm} .2020 .4526$

\begin{abstract}
Lung adenocarcinoma (LUAD) is one of the most common types of lung cancer and its poor prognosis largely depends on the tumor pathological stage. Critical roles of microRNAs (miRNAs) have been reported in the tumorigenesis and progression of lung cancer. However, whether the differential expression pattern of miRNAs could be used to distinguish early-stage (stage I) from mid-late-stage (stages II-IV) LUAD tumors is still unclear. In this study, clinical information and miRNA expression profiles of patients with LUAD were downloaded from The Cancer Genome Atlas (TCGA) and Gene Expression Omnibus databases. TCGA-LUAD $(n=470)$ dataset was used for model training and validation, and the GSE62182 $(n=94)$ and GSE83527 $(n=36)$ datasets were used as external independent test datasets. The diagnostic model was created through miRNA feature selection followed by SVM classifier and was confirmed by 5 -fold cross-validation. A receiver operating characteristic curve was calculated to evaluate the accuracy and robustness of the model. Using the DX score and LIBSVM tool, a 16-miRNA signature that could distinguish LUAD pathological stages was identified. The area under the curve rates were 0.62 [95\% confidence interval (CI): 0.56-0.67], 0.66 (95\% CI: 0.54-0.76) and 0.63 (95\% CI: 0.43-0.82) in TCGA-LUAD internal validation dataset, the GSE62182 external validation dataset, and the GSE83527
\end{abstract}

Correspondence to: Dr Hongkun Yin, Shanghai Yitu Healthcare Technology Co. Ltd., 523 Loushanguan Road, Changning, Shanghai 200051, P.R. China

E-mail: hongkun.yin@yitu-inc.com

Professor Xiaohua Qian, SJTU-Yitu Joint Laboratory of Artificial Intelligence in Healthcare, School of Biomedical Engineering, Shanghai Jiao Tong University, 1954 Huashan Road, Xuhui, Shanghai 200030, P.R. China

E-mail: xiaohua.qian@sjtu.edu.cn

${ }^{*}$ Contributed equally

Key words: lung adenocarcinoma, microRNA signature, pathological stage, diagnosis external validation dataset, respectively. Kyoto Encyclopedia of Genes and Genomes and Gene Ontology enrichment analyses suggested that the target genes of the 16-miRNA signature were mainly involved in metabolic pathways. The present findings demonstrate that a 16-miRNA signature could serve as a promising diagnostic biomarker for pathological staging in LUAD.

\section{Introduction}

Lung cancer is the leading cause of cancer-associated deaths worldwide and has a low 5-year survival rate after diagnosis $(1,2)$. Non-small cell lung cancer (NSCLC) accounts for $~ 85 \%$ of all lung cancer cases and lung adenocarcinoma (LUAD) is the predominant histological subtype of NSCLC, which is often exhibited by females and people who have never smoked (3-5). The poor patient survival rate of NSCLC is primarily due to the high frequency of late diagnosis (6) and the prognosis of NSCLC largely depends on the tumor stage. The lung tumor of patients with NSCLC with pathological stage I disease (early stage) can be completely removed through surgical resection and these patients, therefore, have a 5-year survival rate of $>70 \%$. However, mid-late-stage (stages II-IV) lung cancer is difficult and often impossible to remove completely with surgery, and the 5-year survival rate for patients with stage II-IV disease ranges from 40 to $<10 \%(7,8)$. Thus, accurate staging is critical for NSCLC treatment.

MicroRNAs (miRNAs or miRs) are a class of small ( 22 nucleotides), often phylogenetically conserved noncoding RNAs that are widely expressed and regulate the majority of biological functions (9). Mammalian miRNA binding sites are most commonly found in introns or the $3^{\prime}$ untranslated region of mRNAs (10). After miRNAs are cleaved and activated by the Dicer complex, the activated miRNAs bind to a complementary sequence in the $3^{\prime}$ untranslated region of the target mRNAs, which results in decreased gene expression through translational repression and mRNA destabilization and degradation (11). Since miRNAs regulate gene expression through incomplete base pairing, each miRNA has the ability to regulate multiple genes. Unlike mRNAs, miRNAs are stable and can be easily detected in archived formalin-fixed paraffin-embedded specimens (12). Deregulation of miRNA expression has been linked to the majority of cellular functions, especially those 
involved in cancer initiation and progression (13), making miRNAs attractive biomarkers for the detection, classification, and prognosis of multiple cancer types (14-16).

Previous studies have attempted to identify miRNA signatures as potential biomarkers for patients with lung cancer. Patnaik et al (17) reported a 6-miRNA-based classifier that could predict the recurrence of localized stage I NSCLC based on the miRNA expression profiles of 77 surgically treated pathologic stage I NSCLC cases. Bishop et al (18) reported that a miRNA-based method could be used to classify lung squamous cell carcinoma and LUAD. Li et al (19) identified an 8-miRNA signature as a potential biomarker for predicting survival in LUAD. Although several miRNAs have been identified as predictors of clinical diagnosis or outcome in lung cancer, due to the small patient number and lack of external validation, the models predicted in these studies (17-19) might not be reliable. Importantly, whether miRNAs can be used as pathological staging markers remains unclear. Therefore, a larger patient cohort and an external independent validation cohort for investigation of LUAD staging-specific classifiers are urgently required.

The Cancer Genome Atlas (TCGA) database provides a collection of clinical data, DNA/RNA sequences and DNA methylation profiles of $\geq 500$ cases of 20 different tumor types, which is publicly available (20), while the Gene Expression Omnibus (GEO) is a public functional genomics data repository supporting minimum information about a microarray experiment-compliant data submissions (21). TCGA and GEO contain extensive genomic data, including miRNA sequencing (miRNAseq) data and related clinical information of LUAD cases. Yerukala Sathipati and Ho reported an 18-miRNA signature associated with LUAD patient survival based on the TCGA-LUAD dataset (22). This study used miRNAseq expression profiles downloaded from TCGA and GEO to identify the differential miRNA expression patterns in samples from patients with early and mid-late pathological stage LUAD. Additionally, a 16-miRNA signature that could distinguish early-stage LUAD from mid-late-stage tumor, was constructed. Furthermore, Kyoto Encyclopedia of Genes and Genomes (KEGG) and Gene Ontology (GO) analyses were performed to understand the biological pathways regulated by the prognostic miRNA signature.

\section{Materials and methods}

miRNA expression data collection and preprocessing. The miRNA expression profiles and clinical information from TCGA-LUAD dataset were downloaded from TCGA database (https://portal.gdc.cancer.gov/), while raw data from the GSE62182 (23) and GSE83527 (24) datasets were downloaded from the GEO database (https://www.ncbi.nlm.nih. gov/geo). The expression profiles of the 3 miRNA miRNAseq datasets used in the present study were all generated by an Illumina HiSeq 2000 platform (Illumina, Inc.). These full clinical datasets were assessed for eligibility and nontumor samples (45 cases in TCGA-LUAD, 94 cases in GSE62182 and 41 cases in GSE83527), recurrent tumor samples (2 cases in TCGA-LUAD) and samples lacking staging information ( 7 cases in TCGA-LUAD) were removed. There were 470 available primary LUAD samples with histopathological information in TCGA-LUAD dataset and 94 and 36 available primary LUAD samples in the GSE62182 and GSE83527 datasets, respectively. Notably, 10 LUAD samples from the GSE83527 dataset were originally classified using the Tumor-Node-Metastasis (TNM) staging system and were therefore reclassified following the 8th edition TNM stage classification guide developed by the International Association for the Study of Lung Cancer (25). All the clinical information of the selected samples is summarized in Table I.

The miRNA expression levels were reported as reads per million miRNA mapped (RPM). Since a number of miRNAs were differentially expressed by tumor subtype and differences in sample procurement were observed, the RNA extraction quality, enzymatic efficiency, and other sources might lead to systematic variability. Consequently, the accuracy of the methods used for expression analysis was critically dependent on the proper normalization of the raw data. An ideal normalizer would be a single miRNA that is stable and has invariant expression across all samples. In this study, Homo sapiens (hsa)-miR-191 was used as the normalizer because it was the most stable single miRNA and has been shown to have the lowest expression variability in lung cancer tissues (26). Finally, the hsa-miR-191-normalized RPM values were used to represent the miRNA expression levels in TCGA-LUAD, GSE62182 and GSE83527 datasets.

miRNA selection and model construction. The goal of the miRNA feature selection is to remove redundant features and to identify the most relevant features, thereby improving the classification performance for early-stage and mid-late-stage lung cancer. The expression heatmaps of the selected miRNAs were generated using HemI v1.0, a toolkit for illustrating heatmaps (http://hemi.biocuckoo.org). The present group previously developed a feature selection scheme based on a DX score and successfully evaluated its effectiveness in a classification system $(27,28)$. Briefly, the DX score is used to measure the diversity between positive and negative classes for each feature. The DX scores can be defined as follows: $\mathrm{DX}=\left(\mathrm{m}_{1}-\mathrm{m}_{0}\right)^{2} / \mathrm{d}_{1}^{2}+\mathrm{d}_{0}{ }^{2}+\sigma$, where $m_{1}\left(m_{0}\right)$ and $d_{1}\left(d_{0}\right)$ are the mean value and standard deviation of a feature in a positive (negative) sample, respectively. To avoid a denominator equal to zero when both classes had constant features, a small positive number, namely $\sigma$, was added. The larger the DX score, the better the performance of differentiating between the positive and negative samples by this feature.

Starting with the individual DX scores, the DX score of each feature was ranked from high to low in order to form a ranked feature set and its classification performance was evaluated by 5 -fold cross-validation (CV). This procedure yielded a curve of $\mathrm{CV}$ accuracy with several top-ranked miRNAs. The optimized miRNAs with the best accuracy were identified for later training and testing.

The predictive model was established using the miRNA feature selection scheme based on the DX score and a support vector machine (SVM) classifier. The top-ranked miRNAs with the best accuracy were identified as the optimized miRNAs that could capture the subtle difference between early-stage and mid-late-stage lung cancer. This feature selection scheme was confirmed by 5 -fold $\mathrm{CV}$ and area under the curve (AUC) analysis. In 5-fold $\mathrm{CV}$, the dataset was randomly divided into 
Table I. Demographic and histopathological data of patients from TCGA and GEO databases.

\begin{tabular}{|c|c|c|c|}
\hline Variable & $\begin{array}{l}\text { TCGA-LUAD }(\mathrm{n}=470) \\
\text { Training and validation dataset }\end{array}$ & $\begin{array}{l}\text { GSE62182 }(\mathrm{n}=94) \\
\text { Test dataset } 1\end{array}$ & $\begin{array}{l}\text { GSE83527 }(n=36) \\
\text { Test dataset } 2\end{array}$ \\
\hline \multicolumn{4}{|l|}{ Sex, n (\%) } \\
\hline Female & $255(54.3)$ & $65(69.1)$ & $15(41.7)$ \\
\hline Male & $215(45.7)$ & $29(30.9)$ & $21(58.3)$ \\
\hline \multicolumn{4}{|l|}{ Smoking, n (\%) } \\
\hline Current or former & $320(68.1)$ & $67(71.3)$ & 33 (91.7) \\
\hline Never & $150(31.9)$ & 27 (28.7) & $3(8.3)$ \\
\hline \multicolumn{4}{|l|}{ Stage, n (\%) } \\
\hline I & $260(55.3)$ & $58(61.7)$ & $15(41.6)$ \\
\hline II & $109(23.2)$ & $23(24.5)$ & $15(41.6)$ \\
\hline III & $78(16.6)$ & $10(10.6)$ & $5(13.9)$ \\
\hline IV & $23(4.9)$ & $3(3.2)$ & $1(2.8)$ \\
\hline
\end{tabular}

5 subsets with $\sim$ the same size and each subset had practically the same number of cases of the two types (i.e., early-stage and mid-late-stage lung cancer). Then, 4 subsets were used as the training data and the remaining subset was used to validate the trained classifier. This process was repeated 5 times and each subset was used as the validation data once. The accuracy of the 5-fold $\mathrm{CV}$ was defined as the average classification accuracy over the 5 rounds of validation.

The SVM algorithm (29) was selected for classification since its superior performance is well established theoretically and practically. In addition, SVM is a typical supervised machine learning approach and is employed as a classifier in this predictive model. For most classification and prediction systems, SVM is superior to other machine learning methods, including the neural network and decision tree classifiers (30).

More specifically, a well-established SVM tool, LIBSVM (31), was selected as the classifier. The radial basis function (RBF) was employed as the Kernel function based on various trials. A grid search was also implemented on the RBF parameter $\gamma$ and the trade-off coefficient $C$. To evaluate the accuracy and robustness of each classifier, a receiver operating characteristic (ROC) curve for sensitivity and specificity was calculated. Sensitivity was determined by $\mathrm{TP} /(\mathrm{TP}+\mathrm{FN})$, while specificity was computed by TN/(FP+TN), where TP, FP, FN and $\mathrm{TN}$ refer to true positive, false positive, false negative and true negative, respectively.

GO and KEGG pathway analyses. To investigate the significantly enriched functions of the differentially expressed genes regulated by the miRNAs and to better understand the significant pathways in which the differentially expressed genes were involved, both GO and KEGG analyses were performed by DIANA-miRPath v3.0 online software (32). Predicted interactions between target genes and biological pathways regulated by the miRNAs were identified using DIANA-TarBase v7.0, enabling an experimentally supported miRNA functional annotation (33). Two-sided Fisher's exact test was used to analyze the significance of the GO category and KEGG pathway enrichment, and corrected $\mathrm{P}<0.05$ was considered to indicate a statistically significant difference.

\section{Results}

Study design. Although patients with stage I or stage II LUAD could both be removed by surgery, their 5-year survival rate was different. According to previous reports, stage I LUAD patients had a 5-year survival rate of $>70 \%$, whereas the 5-year survival rate of stage II patients was only $\sim 40 \%(7,8)$. Thus, the aim of the present study was to separate stage I from stage II-IV LUAD, which was based on the 5-year survival rate above or below $50 \%$. In the present study, miRNA expression profiles and tumor staging information were obtained from 3 public datasets that had been sequenced using the same miRNAseq platform (TCGA-LUAD, GSE62182 and GSE83527). Only LUAD tissues with pathological staging information were used in this study. TCGA-LUAD dataset originally contained 528 miRNA profiles of patients with LUAD, but 2 recurrent tumor profiles, 45 solid tissue normal profiles, 4 repeated profiles and 7 profiles without staging information were removed according to the exclusion criteria. To minimize unwanted variation between different datasets, the miRNA expression levels in each profile were normalized to that of hsa-miR-191. The miRNA signature associated with LUAD pathological grade was first trained and validated through the normalized miRNA expression profiles of the remaining 470 patients in TCGA-LUAD dataset. Since the miRNA signature might be overfitted to the training dataset, it was further evaluated in patients from the GSE62182 and GSE83527 independent datasets to test the robustness of the diagnostic model. The study flow diagram is shown in Fig. 1.

miRNA selection, model training and validation. Certain miRNA features were selected based on the SVM method as described above. The top 50 LUAD staging-related miRNAs were selected and it was observed that the combination of the expression levels of the top 42 miRNAs produced the best model for LUAD staging (Fig. 2A). The AUC was used to evaluate the diagnostic ability of the miRNA signature. The ROC curve for pathological diagnosis of LUAD was plotted based on miRNA expression levels and the AUC curve for the signature comprising the 42 miRNAs in the internal 


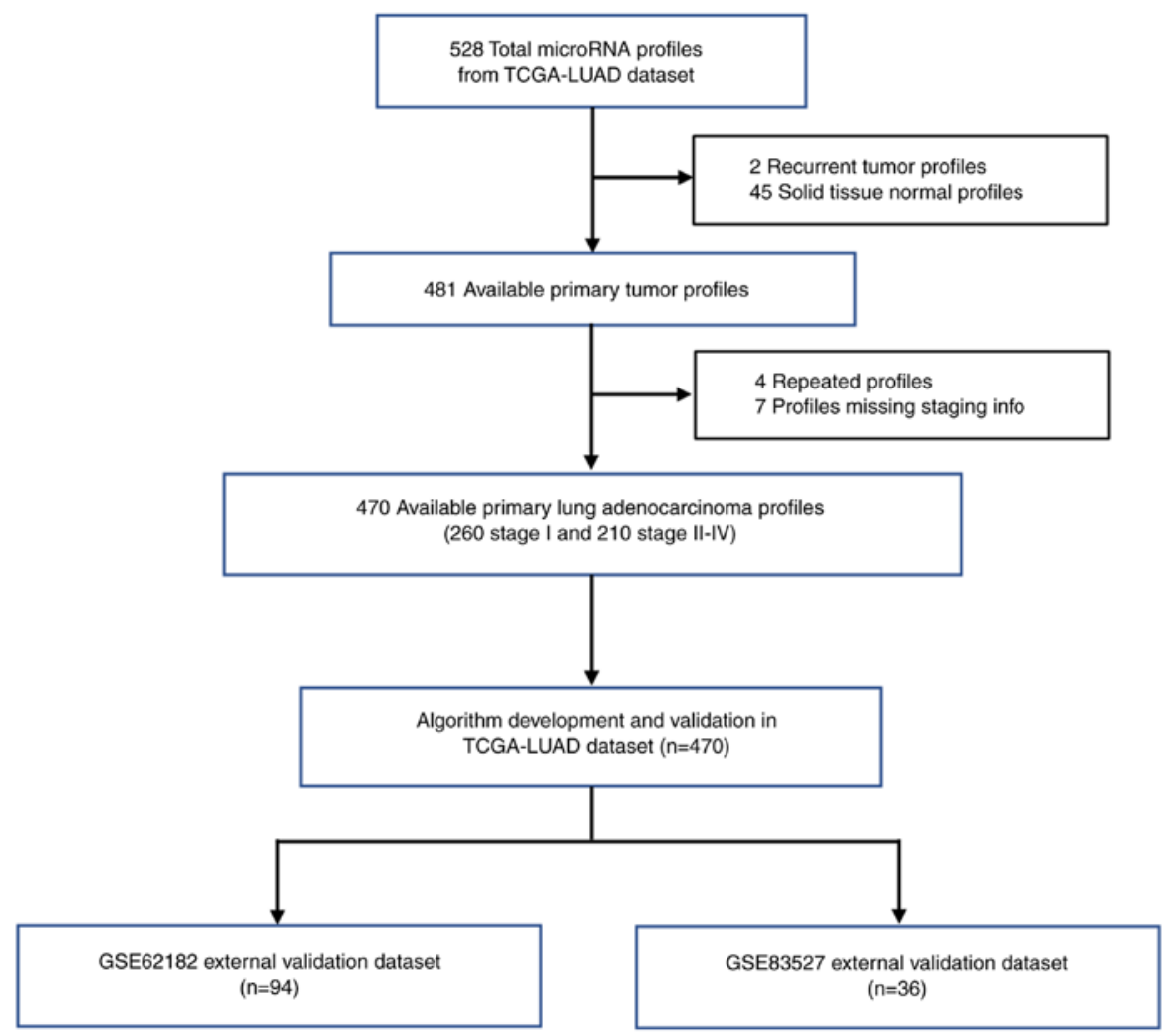

Figure 1. Study flow diagram. The microRNA signature was first generated and internal validated in TCGA-LUAD dataset, and then externally validated in the GSE62182 and GSE83527 datasets to demonstrate its performance. TGCA, The Cancer Genome Atlas; LUAD, lung adenocarcinoma.
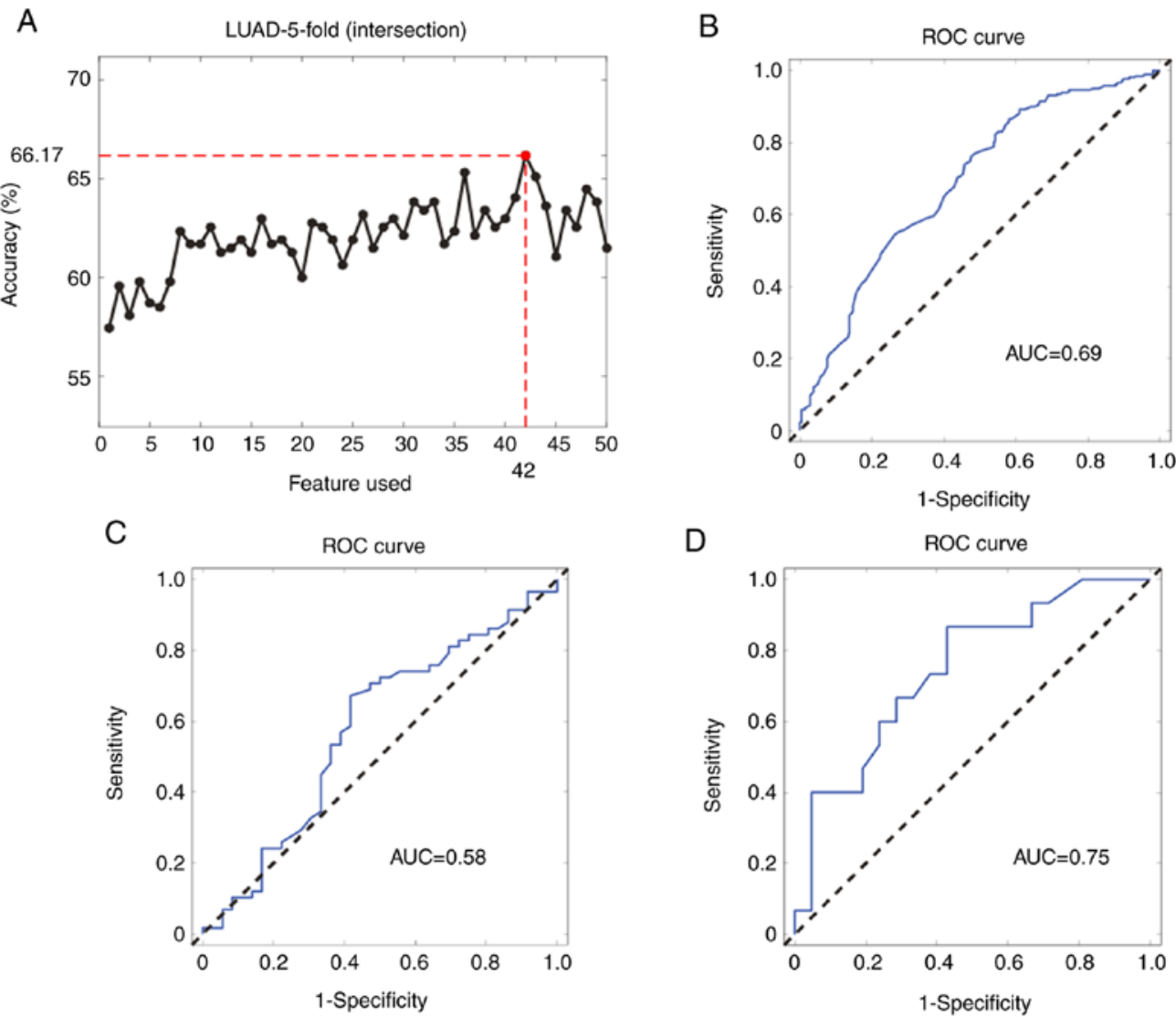

Figure 2. Generation and validation of the 42-miRNA signature. (A) Accuracy curve obtained by selecting the top 50 miRNA features. (B) ROC curve for the internal validation of the 42-miRNA signature in The Cancer Genome Atlas-LUAD dataset. (C) ROC curve for the external validation of the 42-miRNA signature in the GSE62182 dataset. (D) ROC curve for the external validation of the 42-miRNA signature in the GSE83527 dataset. miRNA, microRNA; ROC, receiver operating characteristic; AUC, area under the curve; LUAD, lung adenocarcinoma. 

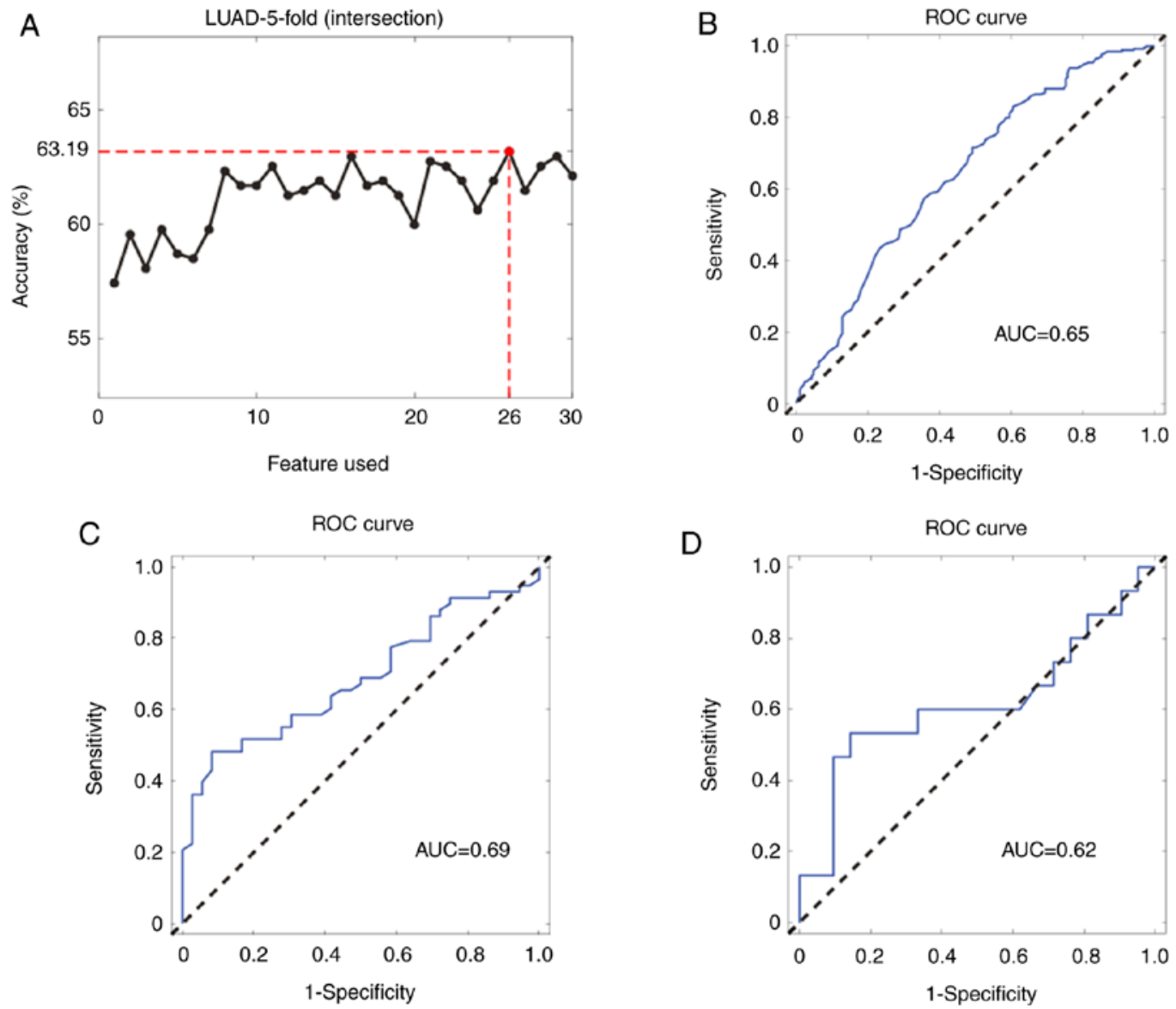

Figure 3. Generation and validation of the 26-miRNA signature. (A) Accuracy curve obtained by selecting the top 30 miRNA features. (B) ROC curve for the internal validation of the 26-miRNA signature in The Cancer Genome Atlas-LUAD dataset. (C) ROC curve for the external validation of the 26-miRNA signature in the GSE62182 dataset. (D) ROC curve for the external validation of the 26-miRNA signature in the GSE83527 dataset. miRNA, microRNA; ROC, receiver operating characteristic; AUC, area under the curve; LUAD, lung adenocarcinoma.

validation dataset was 0.69 [95\% confidence interval (CI): 0.64-0.73] (Fig. 2B). The 42-miRNA model showed a similar performance in the other two external validation datasets [AUC, 0.75 (95\% CI: 0.56-0.88) and 0.58 (95\% CI: 0.45-0.69), respectively; Fig. 2C and D].

However, the combination of 42 miRNAs was markedly complex and could be difficult to use for clinical detection; thus, the accuracy of the combination of other miRNAs to obtain a more simplified miRNA signature was calculated. After permutation and combination analyses, the number of miRNAs was first reduced to 26 . The results showed that the 26-miRNA signature had a similar performance compared to that of all 42 miRNAs (Fig. 3A), with the AUCs in the internal validation dataset and the two independent external validation datasets calculated to be 0.65 (95\% CI: 0.65-0.69), 0.69 (95\% CI: 0.56-0.78) and 0.62 (95\% CI: 0.40-0.82), respectively (Fig. 3B-D).

Then, the number of miRNAs was further reduced to construct an easier and suitable model that could be a potential biomarker for the staging of LUAD. It was observed that the 16-miRNA signature (hsa-mir-2116, hsa-mir-4161, hsa-mir-3942, hsa-mir-4435, hsa-mir-1307, hsa-mir-1254, hsa-mir-582, hsa-mir-5690, hsa-mir-4713, hsa-mir-1293, hsa-mir-939, hsa-mir-421, hsa-mir-335, hsa-mir-4677, hsa-mir-4754 and hsa-mir-4746; Table II) showed a similar ability to classify LUAD pathological stages to that of the combinations of 42 or 26 miRNAs (Fig. 4A). The AUC for the 16-miRNA signature was $0.62(95 \% \mathrm{CI}$ : $0.65-0.67)$ in the internal validation dataset (Fig. 4B), 0.66 (95\% CI: 0.54-0.76) in the GSE62182 external validation dataset (Fig. 4D) and 0.63 (95\% CI: 0.43-0.82) in the GSE83527 external validation dataset (Fig. 4F). The expression heatmaps of the 16 miRNAs in TCGA-LUAD, GSE62182 and GSE83527 datasets were generated using HemI v1.0 software (34) (Fig. 4C, E and G).

$K E G G$ and $G O$ analyses. Since the underlying molecular biology of different stages of LUAD is still not very clear, the present study used KEGG signaling pathway analysis and GO enrichment analysis to better understand the potential biological function and mechanism of the 16-miRNA signature. By selecting $\mathrm{P}<0.05$ as the cut-off criterium in the KEGG pathway analysis, several comprehensive biological pathways regulated by the 16-miRNA signature were revealed in different stages of LUAD, including 'fatty acid biosynthesis' (hsa00061), 'fatty acid metabolism' (hsa01212), 'other glycan degradation' (hsa00511), 'steroid biosynthesis' (hsa00100), 'biosynthesis of unsaturated fatty acids' (hsa01040) and 'ECM-receptor interaction' (hsa04512) (Table III), most of which are metabolic pathways involving biosynthesis, metabolism, degradation of fatty acids and other glycans. Therefore, 
Table II. List of the 16 miRNAs in the signature.

\begin{tabular}{lll}
\hline miRNA ID & miRNA region & Mature miRNA \\
\hline hsa-mir-2116 & MIMAT0011161 & hsa-miR-2116-3p \\
hsa-mir-4661 & MIMAT0019729 & hsa-miR-4661-5p \\
hsa-mir-3942 & MIMAT0018358 & hsa-miR-4435 \\
hsa-mir-4435 & MIMAT0018951 \\
hsa-mir-1307 & MIMAT0005951 & hsa-miR-1307-3p \\
hsa-mir-1254 & MIMAT0005905 & hsa-miR-1254 \\
hsa-mir-582 & MIMAT0004797 & hsa-miR-582-3p \\
hsa-mir-5690 & MIMAT0022482 & hsa-miR-5690 \\
hsa-mir-4713 & MIMAT0019821 & hsa-miR-4713-3p \\
hsa-mir-1293 & MIMAT0005883 & hsa-miR-1293 \\
hsa-mir-939 & MIMAT0004982 & hsa-miR-939-5p \\
hsa-mir-421 & MIMAT0003339 & hsa-miR-421 \\
hsa-mir-335 & MIMAT0000765 & hsa-miR-335-5p \\
hsa-mir-4677 & MIMAT0019760 & hsa-miR-4677-5p \\
hsa-mir-4754 & MIMAT0019894 & hsa-miR-4754 \\
hsa-mir-4746 & MIMAT0019880 & hsa-miR-4746-5p \\
\hline
\end{tabular}

mir/miRNA, microRNAs.

Table III. Enriched biological pathways identified in a KEGG pathway analysis.

\begin{tabular}{|c|c|c|c|c|}
\hline No. & KEGG pathway & P-value & Genes & miRNAs \\
\hline 1 & Fatty acid biosynthesis (hsa00061) & $<1 \times 10^{-325}$ & 1 & 4 \\
\hline 2 & Fatty acid metabolism (hsa01212) & $<1 \times 10^{-325}$ & 6 & 4 \\
\hline 3 & Other glycan degradation (hsa00511) & $9.86 \times 10^{-06}$ & 3 & 2 \\
\hline 4 & Steroid biosynthesis (hsa00100) & $6.13 \times 10^{-05}$ & 13 & 2 \\
\hline 5 & Biosynthesis of unsaturated fatty acids (hsa01040) & 0.01643741 & 4 & 2 \\
\hline 6 & ECM-receptor interaction (hsa04512) & 0.04934272 & 26 & 1 \\
\hline
\end{tabular}

KEGG, Kyoto Encyclopedia of Genes and Genomes; ECM, extracellular matrix; miRNA, microRNA.

these 16 miRNAs might be involved in fatty acid metabolism, indicating that metabolic pathways could play an important role in LUAD progression. The significant categories according to GO results included 'biological process' (Table IV), 'cellular component' (Table V) and 'molecular function' (Table VI). In these categories, various functions associated with RNA processing, gene expression and multiple catabolic/metabolic processes were identified. The clustered heatmap analysis of the enriched KEGG pathways and GO categories was generated by DIANA-miRPath v3.0 using the default settings (Fig. 5). The results indicated that the 16 miRNA classifiers might participate in the development of LUAD through the regulation of a series of pathways, particularly metabolism-related pathways.

\section{Discussion}

Considering that abnormal miRNA expression affects the molecular functions and biological processes of multiple tumors, numerous attempts have been made to use miRNAs as biomarkers for accurate prediction of lung cancer diagnosis and prognosis $(18,22,35)$. However, most previous studies have focused on a small patient sample size. Moreover, most reported lung cancer subtype models do not involve an external confirmation dataset, therefore weakening the reliability of the results. In other words, the miRNA signature might correctly classify tumor status based on one particular dataset but might misclassify other samples from another dataset that are likely to have a different group of patients.

In this study, TCGA-LUAD, one of the largest miRNA expression datasets of LUAD, was selected to establish the current prediction model. Additionally, miRNA profiles from GEO62182 and GEO83527 were used as independent external test datasets for testing the robustness of the present algorithm. The current model showed stable diagnostic capability, as the model achieved similar accuracy in different datasets. However, the classification performance was not very high, probably due to the difficulty of the specific 
A

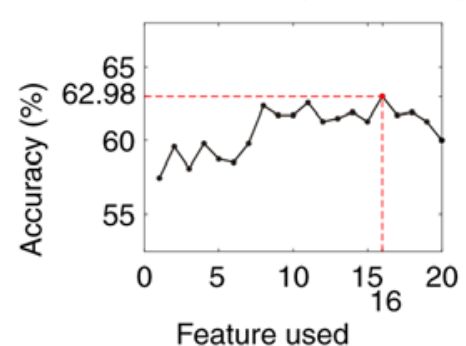

B

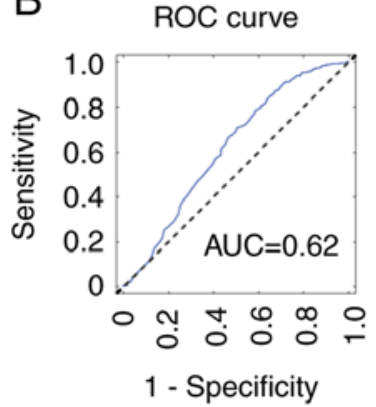

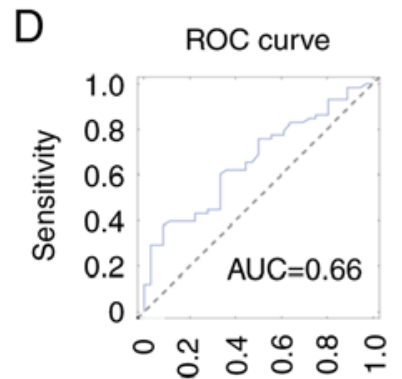

1 - Specificity

C
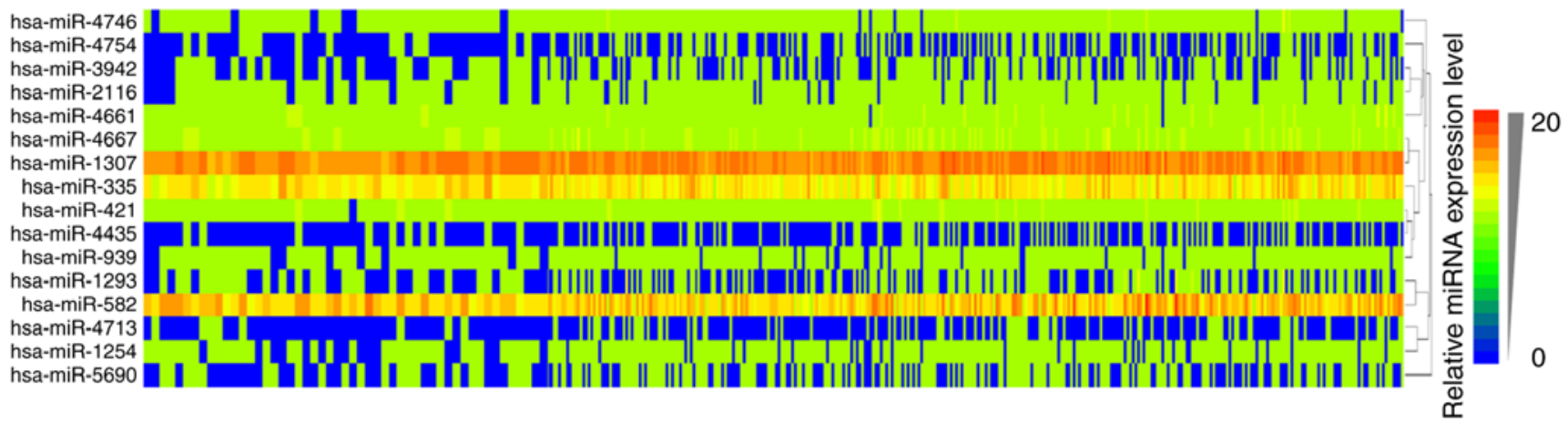

$\mathrm{E}$

Early stage (stage I)

Mid-late stage (stage II IV)
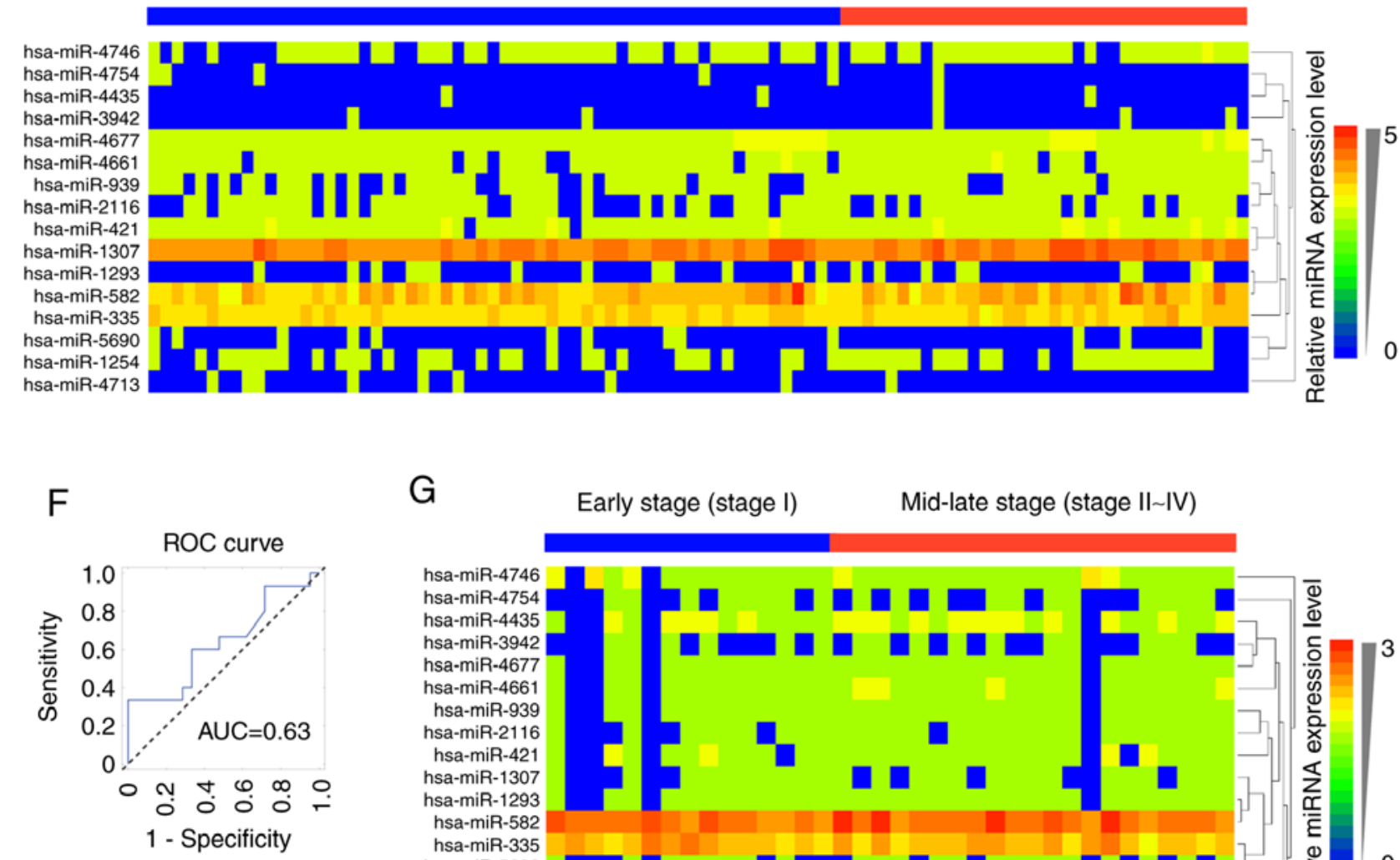

G

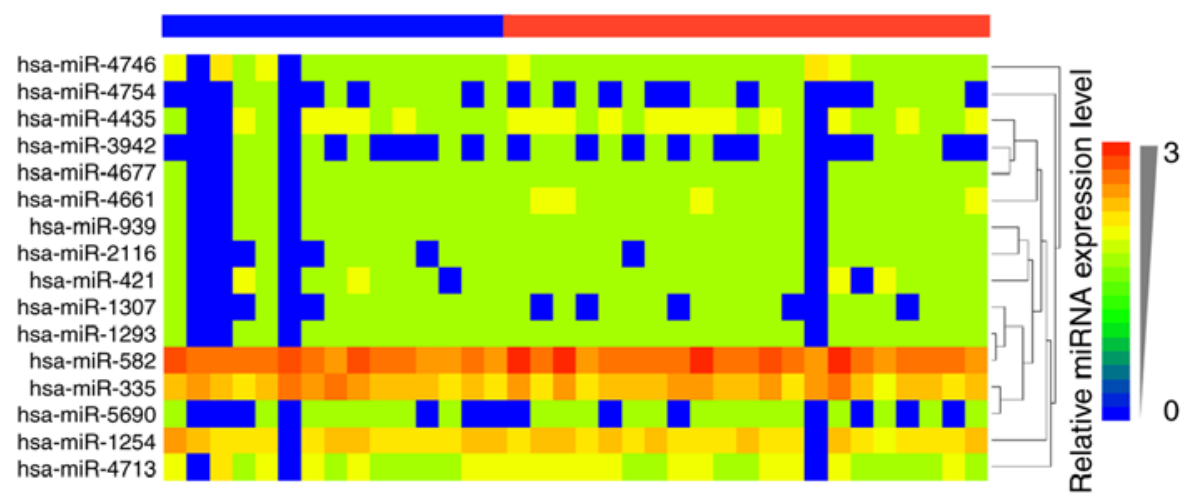

Figure 4. Generation and validation of the 16-miRNA signature. (A) Accuracy curve obtained by selecting the top 20 miRNA features. (B) Classification performance of the 16-miRNA signature in TCGA-LUAD training dataset. (C) Hierarchical clustering of differentially expressed miRNAs in TCGA-LUAD dataset. (D) External validation of the 16-miRNA signature in the GSE62182 dataset. (E) Hierarchical clustering of differentially expressed miRNAs in the GSE62182 dataset. (F) External validation of the 16-miRNA feature in the GSE83527 dataset. (G) Hierarchical clustering of differentially expressed miRNAs in the GSE83527 dataset. TCGA-LUAD, The Cancer Genome Atlas-lung adenocarcinoma; miRNA, microRNA; ROC, receiver operating characteristic; AUC, area under the curve. 
Table IV. List of significant GO terms in the biological process category.

\begin{tabular}{|c|c|c|c|c|}
\hline No. & GO Term (Biological Process) & P-value & Genes & miRNAs \\
\hline 1 & Biological process (GO:0008150) & $<1 \times 10^{-325}$ & 1,353 & 5 \\
\hline 2 & Symbiosis, encompassing mutualism through parasitism (GO:0044403) & $<1 \times 10^{-325}$ & 94 & 5 \\
\hline 3 & Gene expression (GO:0010467) & $<1 \times 10^{-325}$ & 125 & 6 \\
\hline 4 & Biosynthetic process (GO:0009058) & $<1 \times 10^{-325}$ & 450 & 7 \\
\hline 5 & Cellular protein modification process (GO:0006464) & $<1 \times 10^{-325}$ & 562 & 8 \\
\hline 6 & Cellular nitrogen compound metabolic process (GO:0034641) & $<1 \times 10^{-325}$ & 612 & 10 \\
\hline 7 & Viral process (GO:0016032) & $1.11 \times 10^{-16}$ & 85 & 5 \\
\hline 8 & Small molecule metabolic process (GO:0044281) & $1.18 \times 10^{-14}$ & 552 & 5 \\
\hline 9 & Neurotrophin TRK receptor signaling pathway (GO:0048011) & $3.90 \times 10^{-13}$ & 99 & 6 \\
\hline 10 & Nucleobase-containing compound catabolic process (GO:0034655) & $1.04 \times 10^{-12}$ & 126 & 5 \\
\hline 11 & Catabolic process (GO:0009056) & $1.24 \times 10^{-12}$ & 446 & 5 \\
\hline 12 & Cellular protein metabolic process (GO:0044267) & $2.49 \times 10^{-08}$ & 131 & 5 \\
\hline 13 & Response to stress (GO:0006950) & $7.59 \times 10^{-08}$ & 511 & 5 \\
\hline 14 & Blood coagulation (GO:0007596) & $1.66 \times 10^{-06}$ & 115 & 3 \\
\hline 15 & Cell death (GO:0008219) & $1.79 \times 10^{-06}$ & 81 & 3 \\
\hline 16 & Mitotic cell cycle (GO:0000278) & $1.87 \times 10^{-06}$ & 60 & 5 \\
\hline 17 & Membrane organization (GO:0061024) & $1.99 \times 10^{-06}$ & 93 & 5 \\
\hline 18 & Fc-epsilon receptor signaling pathway (GO:0038095) & $5.15 \times 10^{-06}$ & 49 & 3 \\
\hline 19 & mRNA metabolic process (GO:0016071) & $6.09 \mathrm{E}-05$ & 40 & 5 \\
\hline 20 & Epidermal growth factor receptor signaling pathway (GO:0007173) & 0.000209601 & 59 & 3 \\
\hline 21 & Immune system process (GO:0002376) & 0.000331715 & 319 & 3 \\
\hline 22 & RNA metabolic process (GO:0016070) & 0.000428269 & 38 & 4 \\
\hline 23 & Cellular component assembly (GO:0022607) & 0.000625565 & 124 & 4 \\
\hline 24 & RNA splicing (GO:0008380) & 0.002480079 & 35 & 2 \\
\hline 25 & mRNA processing (GO:0006397) & 0.002624066 & 61 & 4 \\
\hline 26 & $\begin{array}{l}\text { Activation of signaling protein activity involved in unfolded protein } \\
\text { response (GO:0006987) }\end{array}$ & 0.01328594 & 22 & 1 \\
\hline 27 & DNA metabolic process (GO:0006259) & 0.02118011 & 54 & 2 \\
\hline 28 & Post-translational protein modification (GO:0043687) & 0.02692964 & 40 & 1 \\
\hline 29 & Transcription, DNA-templated (GO:0006351) & 0.02971434 & 131 & 2 \\
\hline 30 & Fibroblast growth factor receptor signaling pathway (GO:0008543) & 0.04102542 & 50 & 3 \\
\hline
\end{tabular}

GO, gene ontology; miRNA, microRNA.

Table V. List of significant GO terms in the cellular component category.

\begin{tabular}{llrr}
\hline No. & GO Term (Cellular Component) & P-value & Genes \\
\hline 1 & Cellular component (GO:0005575) & $<1 \times 10^{-325}$ & 1,497 \\
2 & Cytosol (GO:0005829) & $<1 \times 10^{-325}$ & 336 \\
3 & Protein complex (GO:0043234) & $<1 \times 10^{-325}$ & 444 \\
4 & Organelle (GO:0043226) & $<1 \times 10^{-325}$ & 2,361 \\
5 & Nucleoplasm (GO:0005654) & $1.99 \times 10^{-13}$ & 158 \\
\hline
\end{tabular}

GO, gene ontology; miRNA, microRNA.

classification task. Unlike distinguishing squamous cell carcinoma from adenocarcinoma, where the two histopathological subtypes arise from different cells with distinct microenvironments (36), there are often more similarities than differences between the miRNA expression patterns of early- and mid-late-stage LUAD. On the other hand, miRNA 

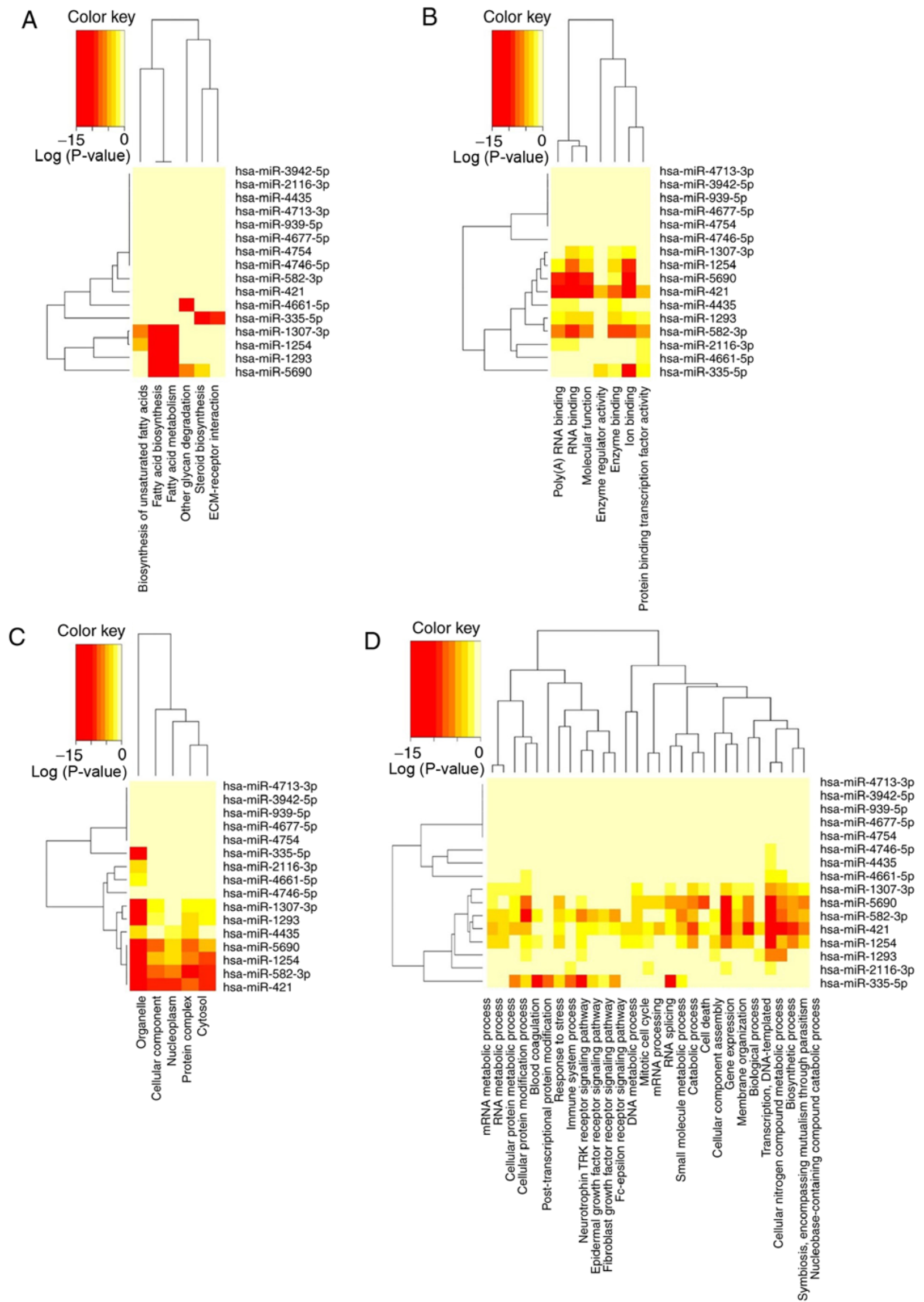

Figure 5. Heatmaps of KEGG pathways and GO annotations of the target genes of the 16 miRNAs in the signature. (A) Heatmap of KEGG pathways from the intersection of the target genes of the 16 miRNAs. The isoforms of the target genes of the 16 miRNAs were involved in multiple pathways, particularly metabolic pathways. (B) Heatmap of GO molecular function from the intersection of the target genes of the 16 miRNAs. (C) Heatmap of GO cellular components from the intersection of the target genes of the 16 miRNAs. (D) Heatmap of GO biological processes from the intersection of the target genes of the 16 miRNAs. KEGG, Kyoto Encyclopedia of Genes and Genomes; GO, Gene Ontology; miRNA, microRNA. 
Table VI. List of significant GO terms in the molecular function category.

\begin{tabular}{llrr}
\hline No. & \multicolumn{1}{c}{ GO Term (Molecular Function) } & P-value & Genes \\
\hline 1 & Molecular function (GO:0003674) & $<1 \times 10^{-325}$ & 1,491 \\
2 & Ion binding (GO:0043167) & $<1 \times 10^{-325}$ & 1,414 \\
3 & Poly(A) RNA binding (GO:0044822) & $<1 \times 10^{-325}$ & 242 \\
4 & RNA binding (GO:0003723) & $<1 \times 10^{-325}$ & 297 \\
5 & Enzyme binding (GO:0019899) & $3.22 \times 10^{-15}$ & 337 \\
6 & Protein binding transcription factor activity (GO:0000988) & $1.03 \times 10^{-07}$ & 8 \\
7 & Enzyme regulator activity (GO:0030234) & 0.02094084 & 131 \\
\end{tabular}

GO, gene ontology; miRNA, microRNA.

expression might only provide limited information for tumor staging. Multi-omics data, including mRNA expression and CpG methylation, could lead to multi-omics integration and help to discover more sensitive molecular features. In liver cancer patient survival prediction, multi-omics data have shown better performance than single-omics data for model building (37).

Among the aforementioned 16 miRNAs, the overexpression of hsa-miR-939 was correlated with poor prognosis in lung cancer (38), as well as promoting epithelial to mesenchymal transition in epithelial ovarian cancer (39). It has been reported that hsa-miR421 promotes tumor progression in hepatocellular carcinoma (40) and osteosarcoma (41), and hsa-miR-335 participates in the progression of gallbladder carcinoma (42). On the other hand, hsa-miR-582 functions as a tumor suppressor in colorectal cancer (43) and hsa-miR-1254 inhibits cell migration and invasion in gastric cancer (44).

Based on the 16-miRNA signature, KEGG and GO analyses were employed to predict the target genes and related pathways, and the results showed that the diagnostic miRNAs regulate metabolic processes such as glycan degradation, fatty acid biosynthesis and metabolism, which is consistent with other reports (45-47). Deregulated metabolism is considered an important hallmark of cancer initiation, progression, metastasis and immune evasion (45). miRNAs are involved in the regulation of cell metabolism, which in turn regulates the molecular mechanisms driving the Warburg effect in cancer cells, including glucose uptake, glycolysis, lipid metabolism and amino acid biogenesis (46). In addition, changes in the tumor environment at different pathological stages could alter the cell metabolism in NSCLC (47). In conclusion, the present results indicate that dysregulation of fatty acid and glycan metabolism might be a critical change during the development of LUAD.

There were several limitations in this study. First, the current research only focused on LUAD, which means that the 16-miRNA pathological staging signature is not suitable for other types of lung cancer, such as squamous cell carcinoma or small cell lung cancer. Second, this study is based on TCGA and GEO public datasets, which are retrospective; thus, the performance of the 16-miRNA signature needs to be validated in future clinical studies. Third, the present diagnostic model only used miRNA expression as single-omics data; thus, incorporating more molecular-omics data such as mRNA expression, $\mathrm{CpG}$ methylation and genomic information might help to improve the accuracy of the model.

In conclusion, the present study identified a novel 16-miRNA signature based on a large sample size and multi-source data, which is promising and effective at predicting the pathological stages of patients with LUAD. In the authors' future studies, multi-omics information should be used to improve the model.

\section{Acknowledgements}

Not applicable.

\section{Funding}

No funding was received.

\section{Availability of data and materials}

All data used in this study can be downloaded from TCGA (https://portal.gdc.cancer.gov/) and GEO (https://www.ncbi. nlm.nih.gov/geo) databases.

\section{Authors' contributions}

HY and XQ designed the study and wrote the manuscript. ZY performed data analyses. LS was responsible for interpretation of the results. XQ and LS provided the resources and supervised the study. All authors have read and approved the final version of the manuscript.

\section{Ethics approval and consent to participate}

Not applicable.

\section{Patient consent for publication}

Not applicable.

\section{Competing interests}

The authors declare that they have no competing interests. 


\section{References}

1. Nanavaty P, Alvarez MS and Alberts WM: Lung cancer screening: Advantages, controversies, and applications. Cancer Control 21: 9-14, 2014.

2. Torre LA, Bray F, Siegel RL, Ferlay J, Lortet-Tieulent J and Jemal A: Global cancer statistics, 2012. CA Cancer J Clin 65: 87-108, 2015

3. Zappa $\mathrm{C}$ and Mousa SA: Non-small cell lung cancer: Current treatment and future advances. Transl Lung Cancer Res 5: 288-300, 2016.

4. Yano T, Haro A, Shikada Y, Maruyama R and Maehara Y: Non-small cell lung cancer in never smokers as a representative 'non-smoking-associated lung cancer': Epidemiology and clinical features. Int J Clin Oncol 16: 287-293, 2011

5. Hsu LH, Chu NM, Liu CC, Tsai SY, You DL, Ko JS, Lu MC and Feng AC: Sex-associated differences in non-small cell lung cancer in the new era: Is gender an independent prognostic factor? Lung Cancer 66: 262-267, 2009.

6. Siegel RL, Miller KD and Jemal A: Cancer statistics, 2019. CA Cancer J Clin 69: 7-34, 2019.

7. Goldstraw P, Chansky K, Crowley J, Rami-Porta R, Asamura H, Eberhardt WE, Nicholson AG, Groome P, Mitchell A and Bolejack V; International Association for the Study of Lung Cancer Staging and Prognostic Factors Committee, Advisory Boards, and Participating Institutions; International Association for the Study of Lung Cancer Staging and Prognostic Factors Committee Advisory Boards and Participating Institutions: The IASLC lung cancer staging project: Proposals for revision of the TNM stage groupings in the forthcoming (eighth) edition of the TNM Classification for lung cancer. J Thorac Oncol 11: 39-51, 2016.

8. Rami-Porta R, Crowley JJ and Goldstraw P: The revised TNM staging system for lung cancer. Ann Thorac Cardiovasc Surg 15 4-9,2009.

9. Oliveto S, Mancino M, Manfrini N and Biffo S: Role of microRNAs in translation regulation and cancer. World $\mathrm{J}$ Biol Chem 8: 45-56, 2017.

10. Shivdasani RA: MicroRNAs: Regulators of gene expression and cell differentiation. Blood 108: 3646-3653, 2006.

11. Felekkis K, Touvana E, Stefanou CH and Deltas C: MicroRNAs: A newly described class of encoded molecules that play a role in health and disease. Hippokratia 14: 236-240, 2010.

12. Xi Y, Nakajima G, Gavin E, Morris CG, Kudo K, Hayashi K and $\mathrm{Ju} \mathrm{J}$ : Systematic analysis of microRNA expression of RNA extracted from fresh frozen and formalin-fixed paraffin-embedded samples. RNA 13: 1668-1674, 2007.

13. MacFarlane LA and Murphy PR: MicroRNA: Biogenesis, function and role in cancer. Curr Genomics 11: 537-561, 2010.

14. Lan H, Lu H, Wang $X$ and Jin H: MicroRNAs as potential biomarkers in cancer: Opportunities and challenges. Biomed Res Int 2015: 125094, 2015.

15. Paranjape T, Slack FJ and Weidhaas JB: MicroRNAs: Tools for cancer diagnostics. Gut 58: 1546-1554, 2009.

16. Grady WM and Tewari M: The next thing in prognostic molecular markers: MicroRNA signatures of cancer. Gut 59: 706-708, 2010.

17. Patnaik SK, Kannisto E, Knudsen $S$ and Yendamuri S: Evaluation of microRNA expression profiles that may predict recurrence of localized stage I non-small cell lung cancer after surgical resection. Cancer Res 70: 36-45, 2010.

18. Bishop JA, Benjamin H, Cholakh H, Chajut A, Clark DP and Westra WH: Accurate classification of non-small cell lung carcinoma using a novel microRNA-based approach. Clin Cancer Res 16: 610-619, 2010

19. Li X, Shi Y, Yin Z, Xue X and Zhou B: An eight-miRNA signature as a potential biomarker for predicting survival in lung adenocarcinoma. J Transl Med 12: 159, 2014

20. Chandran UR, Medvedeva OP, Barmada MM, Blood PD, Chakka A, Luthra S, Ferreira A, Wong KF, Lee AV, Zhang Z, et al: TCGA expedition: A data acquisition and management system for TCGA data. PLoS One 11: e0165395, 2016.

21. Barrett T, Wilhite SE, Ledoux P, Evangelista C, Kim IF, Tomashevsky M, Marshall KA, Phillippy KH, Sherman PM, Holko M, et al: NCBI GEO: Archive for functional genomics data sets-update. Nucleic Acids Res 41: D991-D995, 2013.

22. Yerukala Sathipati S and Ho SY: Identifying the miRNA signature associated with survival time in patients with lung adenocarcinoma using miRNA expression profiles. Sci Rep 7: 7507,2017
23. Vucic EA, Thu KL, Pikor LA, Enfield KS, Yee J, English JC, MacAulay CE, Lam S, Jurisica I and Lam WL: Smoking status impacts microRNA mediated prognosis and lung adenocarcinoma biology. BMC Cancer 14: 778, 2014

24. Becker-Santos DD, Thu KL, English JC, Pikor LA, Martinez VD, Zhang M, Vucic EA, Luk MT, Carraro A, Korbelik J, et al: Developmental transcription factor NFIB is a putative target of oncofetal miRNAs and is associated with tumour aggressiveness in lung adenocarcinoma. J Pathol 240: 161-172, 2016.

25. Rami-Porta R, Bolejack V, Giroux DJ, Chansky K, Crowley J, Asamura $\mathrm{H}$ and Goldstraw P; International Association for the Study of Lung Cancer Staging and Prognostic Factors Committee, Advisory Board Members and Participating Institutions: The IASLC lung cancer staging project: The new database to inform the eighth edition of the TNM classification of lung cancer. J Thorac Oncol 9: 1618-1624, 2014.

26. Peltier HJ and Latham GJ: Normalization of microRNA expression levels in quantitative RT-PCR assays: Identification of suitable reference RNA targets in normal and cancerous human solid tissues. RNA 14: 844-852, 2008.

27. Qian X, Tan H, Zhang J, Zhuang X, Branch L, Sanger C, Thompson A, Zhao W, Li KC, David L and Zhou X: Objective classification system for sagittal craniosynostosis based on suture segmentation. Med Phys 42: 5545-5558, 2015.

28. Tan H, Bao J and Zhou X: A novel missense-mutation-related feature extraction scheme for 'driver' mutation identification. Bioinformatics 28: 2948-2955, 2012.

29. Cortes C and Vapnik V: Support-Vector Networks. Mach Learn 20, 273-297, 1995.

30. You ZH, Yin Z, Han K, Huang DS and Zhou X: A semi-supervised learning approach to predict synthetic genetic interactions by combining functional and topological properties of functional gene network. BMC Bioinformatics 11: 343, 2010.

31. Chang CC and Lin CJ: LIBSVM: A library for support vector machines. ACM Trans Intell Syst Technol, 2011.

32. Vlachos IS, Zagganas K, Paraskevopoulou MD, Georgakilas G, Karagkouni D, Vergoulis T, Dalamagas T and Hatzigeorgiou AG: DIANA-miRPath v3.0: Deciphering microRNA function with experimental support. Nucleic Acids Res 43: W460-W466, 2015.

33. Vlachos IS, Paraskevopoulou MD, Karagkouni D, Georgakilas G, Vergoulis T, Kanellos I, Anastasopoulos IL, Maniou S, Karathanou K, Kalfakakou D, et al: DIANA-TarBase v7.0: Indexing more than half a million experimentally supported miRNA:mRNA interactions. Nucleic Acids Res 43: D153-D159, 2015.

34. Deng W, Wang Y, Liu Z, Cheng H and Xue Y: HemI: A toolkit for illustrating heatmaps. PLoS One 9: e111988, 2014.

35. Saito M, Schetter AJ, Mollerup S, Kohno T, Skaug V, Bowman ED, Mathé EA, Takenoshita S, Yokota J, Haugen A and Harris CC: The association of microRNA expression with prognosis and progression in early-stage, non-small cell lung adenocarcinoma: A retrospective analysis of three cohorts. Clin Cancer Res 17: $1875-1882,2011$.

36. Giangreco A, Groot KR and Janes SM: Lung cancer and lung stem cells: Strange bedfellows? Am J Respir Crit Care Med 175: 547-553, 2007.

37. Chaudhary K, Poirion OB, Lu L and Garmire LX: Deep learning-based multi-omics integration robustly predicts survival in liver cancer. Clin Cancer Res 24: 1248-1259, 2018.

38. Han X, Du C, Chen Y, Zhong X, Wang F, Wang J, Liu C, Li M, Chen S and Li B: Overexpression of miR-939-3p predicts poor prognosis and promotes progression in lung cancer. Cancer Biomark 25: 325-332, 2019.

39. Tang M, Jiang L, Lin Y, Wu X, Wang K, He Q, Wang X and Li W: Platelet microparticle-mediated transfer of miR-939 to epithelial ovarian cancer cells promotes epithelial to mesenchymal transition. Oncotarget 8: 97464-97475, 2017.

40. Wang W, Li Y, Li X, Liu B, Han S, Li X, Zhang B, Li J and Sun S: Circular RNA circ-FOXP1 induced by SOX9 promotes hepatocellular carcinoma progression via sponging miR-875-3p and miR-421. Biomed Pharmacother 121: 109517, 2020.

41. Ren Z, He M, Shen T, Wang K, Meng Q, Chen X, Zhou L, Han Y, Ji C, Liu S and Fu Q: MiR-421 promotes the development of osteosarcoma by regulating MCPIP1 expression. Cancer Biol Ther 21: 231-240, 2020.

42. Wang W, Chen LC, Qian JY and Zhang Q: MiR-335 promotes cell proliferation by inhibiting MEF2D and sensitizes cells to 5-Fu treatment in gallbladder carcinoma. Eur Rev Med Pharmacol Sci 23: 9829-9839, 2019. 
43. Geng Y, Zheng X, Hu W, Wang Q, Xu Y, He W, Wu C, Zhu D, Wu C and Jiang J: Hsa circ 0009361 acts as the sponge of miR-582 to suppress colorectal cancer progression by regulating APC2 expression. Clin Sci (Lond) 133: 1197-1213, 2019.

44. Jiang M, Shi L, Yang C, Ge Y, Lin L, Fan H, He Y, Zhang D, Miao Y and Yang L: MiR-1254 inhibits cell proliferation, migration, and invasion by down-regulating Smurf1 in gastric cancer. Cell Death Dis 10: 32, 2019.

45. Lunt SY and Fendt SM: Metabolism-A cornerstone of cancer initiation, progression, immune evasion and treatment response. Curr Opin Syst Biol 8: 67-72, 2018.

46. Chen B, Li H, Zeng X, Yang P, Liu X, Zhao X and Liang S: Roles of microRNA on cancer cell metabolism. J Transl Med 10: 228, 2012.
47. Davidson SM, Papagiannakopoulos T, Olenchock BA, Heyman JE, Keibler MA, Luengo A, Bauer MR, Jha AK, O'Brien JP, Pierce KA, et al: Environment impacts the metabolic dependencies of ras-driven non-small cell lung cancer. Cell Metab 23: 517-528, 2016.

This work is licensed under a Creative Commons Attribution-NonCommercial-NoDerivatives 4.0 International (CC BY-NC-ND 4.0) License. 